\title{
The Application of Regulating Plant Growth into Protective Engineering of The Western Mansions Area in The Old Summer Palace
}

\author{
Weiwei Cong ${ }^{\mathrm{a}}$, Yingjia Song ${ }^{\mathrm{b}}$ \\ ${ }^{a}$ Senior Designer, Tsinghua Heritage Institute For Digitization, \\ F3\&4,Building1,No.41 Shangdi West Road,Haidian District,Beijing,P.R.China-congweiwei@thid.cn \\ b Senior Designer , Tsinghua Heritage Institute For Digitization, \\ F3\&4,Building1,No.41 Shangdi West Road,Haidian District,Beijing,P.R.China- songyingjia@thid.cn
}

KEY WORDS: Plant Growth; Old Summer Palace; Western Mansions; Relics Protection; heritage ABSTRACT:

The Western Mansions (Xīyáng Lóu) in the Old Summer Palace (Yuánmíngyuán ) are extremely valuable set of heritage. When it was first conceived and constructed, it was an important part of the successful exchange between Chinese and the Western cultures. It marked the entry of European architecture and the art of gardening into Chinese royal gardens. Therefore, the Western Mansions occupies a vital position in the garden history of China and even the world.

Since the 1980s, the Administrative Office of the Old Summer Place (Yuánmíngyuán) had cleared and reorganized the Western Mansions relics. But currently, both the protection and display of the Western Mansions are far from satisfactory. With regards to the outlook of the cultural relics, there is cracking and collapses, relics are damaged by tourists, there is rainwater erosion, plant aggression, pollutants, weathering, crumbling and other threats. This is caused by the improper protective measures and the yearslong erosion through natural causes.

Therefore,in order to protect the important heritage and_show people the situation of the plant growth, As the basis of the copper planting , the historical conditions of planting can be speculated at the time of the western building, which become an important basis for the recovery of history 。

This paper also further researches on the informationg of plant form in history,to avoid destabilising the heritage.Some destruction from plant growth can be controled.

\section{GENERAL INTRODUCTION}

\subsection{Geographical position Instructions}

The Old Summer Palace located in the eastern part of China, Haidian District, west of the Three Hills and Five Gardens Area. It is close to Tsinghua University and Beijing University. (1-1)

The Western Mansions area is located in the northern end of the Old Summer Place (Yuánmíngyuán) .(1-2)

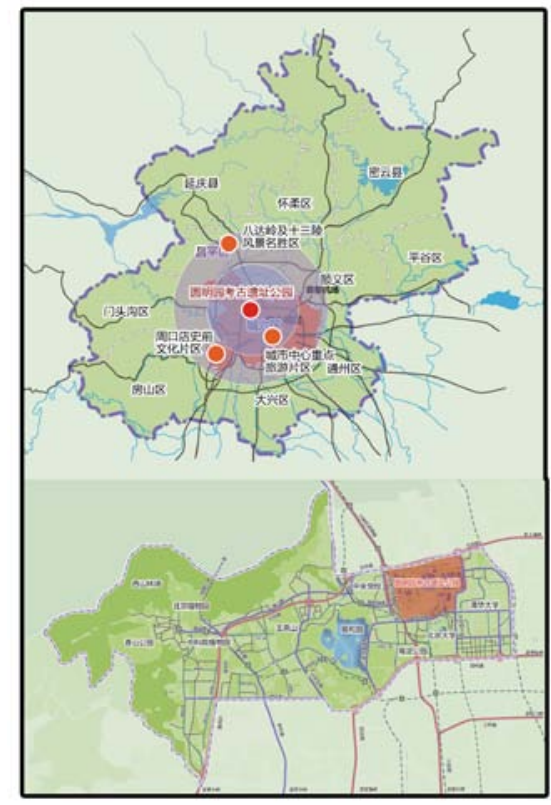

1-1Old Summer Palace in Beijing

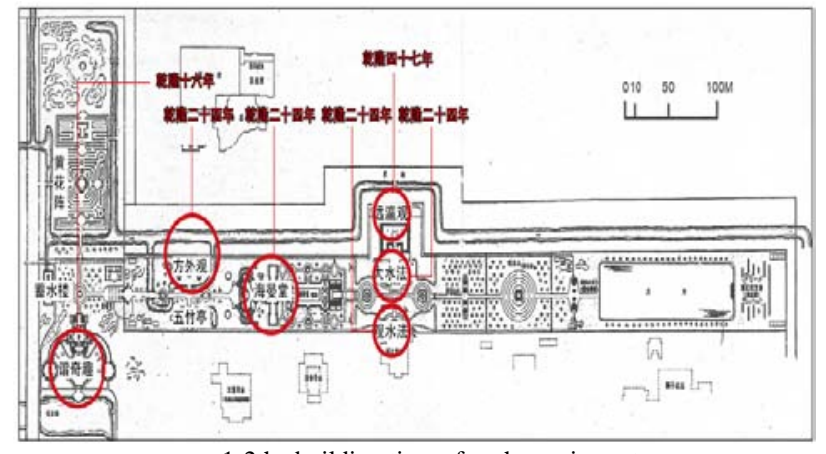

1-2the building time of each scenic spot

\section{ANALYSIS OF INHIBITING PLANT GROWTH IN ANCIENT TIMES}

\subsection{Analysis of inhibiting plant growth in ancient literature}

Eradicate plant breeding via manpower .Since ancient times, it has been a primitive cultivation mode to sow when it is cold and to weed when it is hot. The farming is hard. In addition to sowing, the daily maintenance, namely the weeding, is more important. In the eyes of ancient people, weeding is an essential part of farming. In ancient times, forests were everywhere. Therefore, before sowing, it was a must to cut down trees and remove weeds. There were no metal tools. The only production tools were the polished stone tools, wooden tools and clam shells. Therefore, to cultivate crops, the ancient men would weed grass and loose soils with a clam shell in hand. Both the Dawenkou Culture and the Longshan Culture have unearthed the sickles made from mussel shells. 
Weeding via external force. Weeding with the help of water and fire .Flooding is one of the main abiotic stresses suffered by plants. In regard to weeding, in ancient China, there is a saying that farming by fire and weeding by water. From a scientific perspective, flooding stress will make plants experience a longterm or periodic anaerobic or anoxic condition. This limits the aerobic respiration of plants and the energy production required to maintain life activities. Consequently, thus posing a serious threat to the survival of the plants. Fire is the most extensive cultivation model in primitive farming. Burn weeds and trees, and destroy a piece of original ecology in order to obtain cultivated soil. The ancestors took advantages of the forces of the five elements in nature to weed grass, which reflects their wisdom of life and thinking pattern. Obviously, these methods are not suitable for relic areas.

Weeding via mutual promotion and restraint between the plants.Ancient literature recorded about inhibiting the growth of the plants, such as Mengxi Bitan written by Shen Kuo in the Northern Song dynasty. It is said that the cassia twig debris can be used to remove perennial weeds. Ancient people found that osmanthus sawdust could kill vegetation in the courtyard. If the katsuragi was made into wooden nails and then a nail was forced into the trunk of other trees, the tree would be killed. The ancestors took full advantages of different behaviors and characteristics of plants and found out the law of mutual promotion and restraint between plants. Then, they utilized this law to control or inhibit the growth of plants.

\subsection{Analysis of history plant growth}

As the basis of the copper planting , the historical conditions of planting can be speculated at the time of the western building, which become an important basis for the recovery of history.(21), (2-2), (2-3).

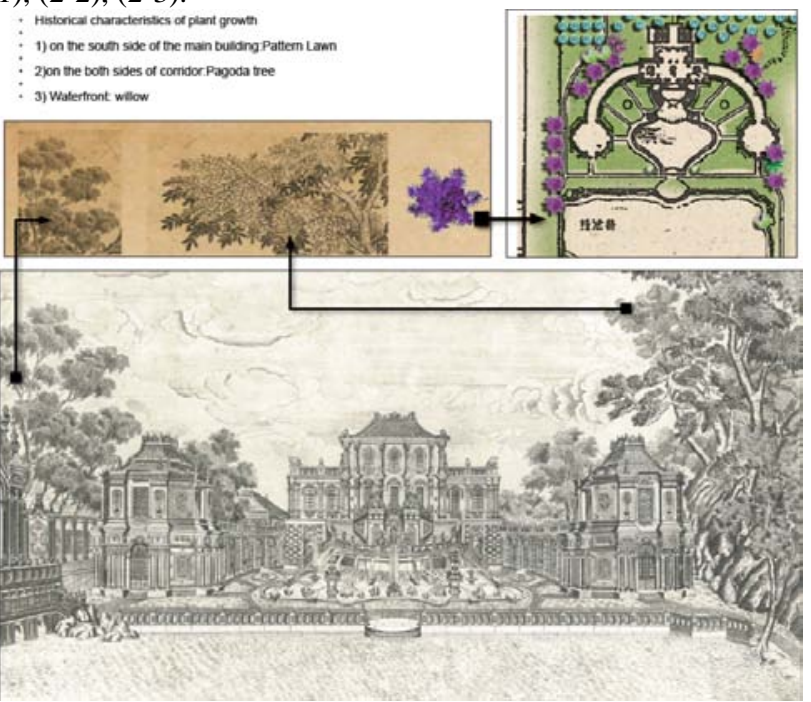

2-1 Harmonic Trolltech sites (the part of The Western Mansions)

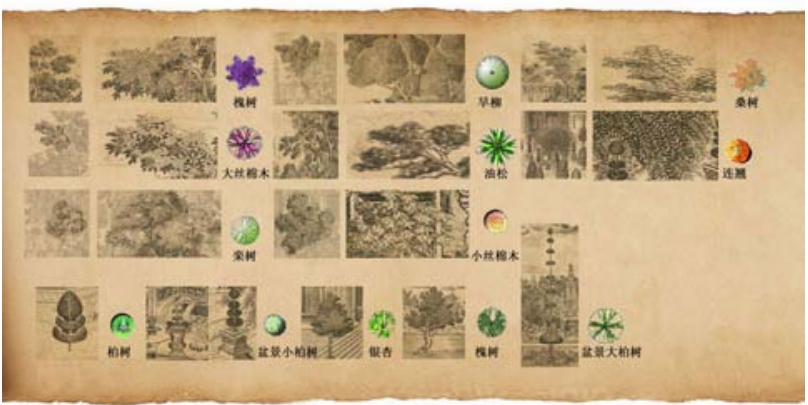

2-2 The plant species, which is inferred from the copper prints

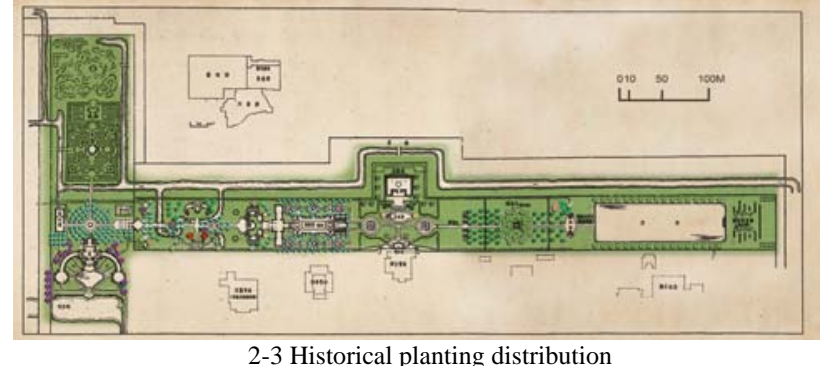

\section{3、PLANNING OF INHIBITING PLANT GROWTH IN WESTERN MANSIONS SCENIC AREA}

\subsection{Plant design principle in Western Mansions scenic area}

3.1.1Rules for plant design in the old Western Mansions scenic area.

The Western Mansions are extremely valuable relics. They were an important part of the exchange between Chinese and the Western cultures. The general principle of vegetation design in the Western Mansions area should treat the protection of related cultural relics as the main direction and inhibit the plants where the relics are damaged, to control the excessive growth of plants. On the basis of the historical investigation of the relics, the historical vegetation restoration research has been completed, with the relics' vegetation in the area as the very object. By doing so, it aims to let the cultural relics be more able to witness the ups and downs of history.

Protect cultural relics and prohibit damage..Resolutely prohibit passive transplanting: most of the vegetation in the park has grown into trees with a developed root system. If they are transplanted, the earth needs to be excavated. According to the general experience, the soil ball diameter of the root is related to the tree. Take the most frequently seen adult locust in the park as an example. The average diameter at breast height is $20 \mathrm{~cm}$, then the size of the soil ball is $1.4-2 \mathrm{~m}$. But the excavation process and the following pit after the excavation will destroy the cultural relics. Therefore, in the process of cleaning up the trees, the passive transplanting should be prohibited. The utilization of chemical agents should be controlled strictly: in the adjustment process of the vegetation, the utilization of chemical agents should be placed under control. The acid, alkali and other chemicals that will damage the cultural relics should be banned. Don't destroy or interfere in the status quo of the cultural relics of the heritage site: if the adult trees and big shrubs which have caused damage to the cultural relics and the site are eradicated from the roots, it may accelerate the cracking of the cultural relics and cause more serious damage. Instead, for these trees and shrubs, only the parts above the ground should be eradicated and before the archaeological excavation work begins, the underground roots systems should not be eradicated. New seedlings and shrubs with small and shallow roots systems can be eradicated directly via physical methods in order not to take out the roots and cause further damage to the cultural relics. For the emerging weeds on the ground, physical methods can be adopted. And during the process of park management, efforts of periodic cleaning should be strengthened. Avoid earth excavation and restore the unique plant morphologies and plant layouts with potted plants. Within the site, the existent plant communities present a natural layout. In the early phase, we only need to rearrange, trim and restore the cypress with regular layout. By doing so, the purpose of displaying the unique historical and artistic value of Western Mansions can be realized easily. After the completion of the archaeological excavation and protection of the main body in 
the whole scenic area of the Western Mansions, the overall adjustment of the park vegetation will be considered, to restore the historical plants landscapes.

Adhere to ecological principle and reduce the destruction of vegetation as much as possibleCurrently in the park, the internal vegetation grows well and the plant community is relatively stable, which has formed favorable ecological benefit. Therefore, every effort should be made so as not to adjust the vegetation and interfere with its growth and cause ecological losses as far as possible. The vegetation which does not damage or interfere in the cultural relics should be kept. Moreover, the vegetation which has damaged or will damage or interfere in the cultural relics should be handled with a mild method. And the parts above ground should be taken out, but the parts under the ground should be kept.

Avoid misinterpreting historical information .Nowadays in the park, there are artificially reseeded trimmed hedges. According to the study of historical materials and copperplate engraving, when the Western Mansions were originally built, it did not adopt the trimmed hedges at all. Moreover, regard to this planting form, both the plants materials and the planting methods are commonly adopted in Classic Western gardens. Therefore, it is probably to cause misinterpretations for the historical information among tourists, thus hindering the display and heritage of such information concerning the Western Mansions. Plants of this kind should be cleaned up.

3.1.2Problems in various sites of scenic areas of the Western Mansions

Because of the lack of regular cleaning in the site, weeds grow in some parts rampantly. Some shrubs and trees have deeply taken root at the site, which will harm the safety of cultural relics. At present, the site damage caused by the vegetation can be specified from the following aspects.

Roots systems of trees: in terms of the trees which grow near the construction ruins or the road ruins, the growth of the roots has caused serious damage to the system of the site and this has led to the cracking or tilting of cultural relics. (3-1),(3-2)

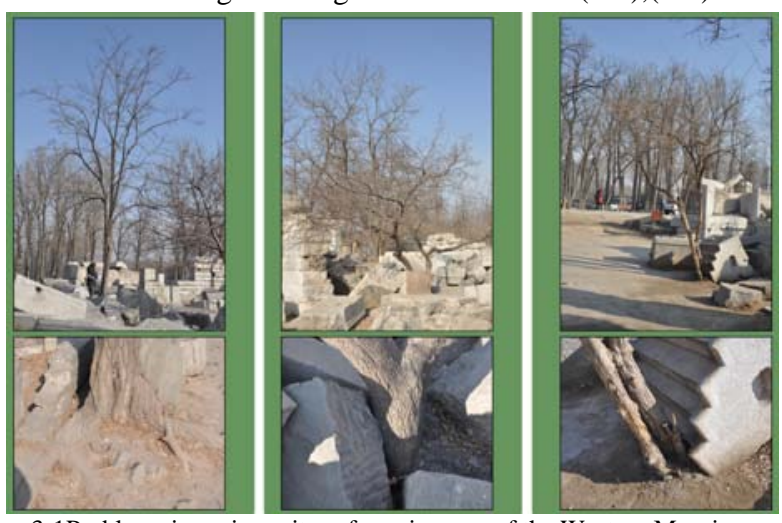

3-1Problems in various sites of scenic areas of the Western Mansions

Bushes: it is likely for the bushy roots of small bushes to attach to the gaps between the cultural relics. Their breeding will lead to the cracking or crumbling of the cultural relics.

Turf and ground cover plant: in the park, the weeds grow so vigorously that they erode and cover the ruins of the roads, which lead to the cracking of the cultural relics. Besides, due to the water collection function of the lawns, a large number of the cultural relics are settled in a moist environment.

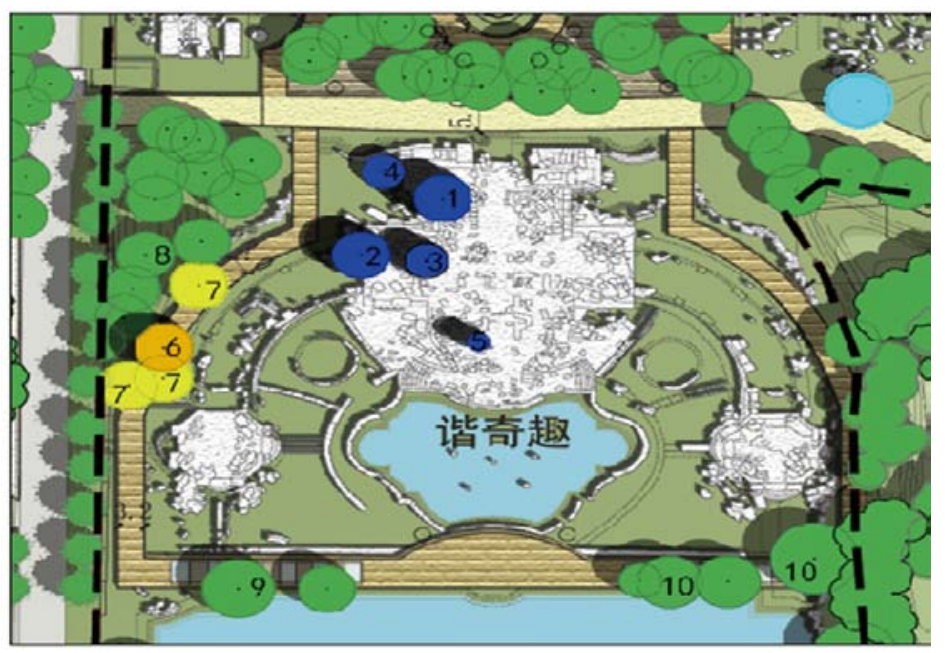

destroy the heritage--clean (5)

stop the passage---clean (1)

pruning branches (3)

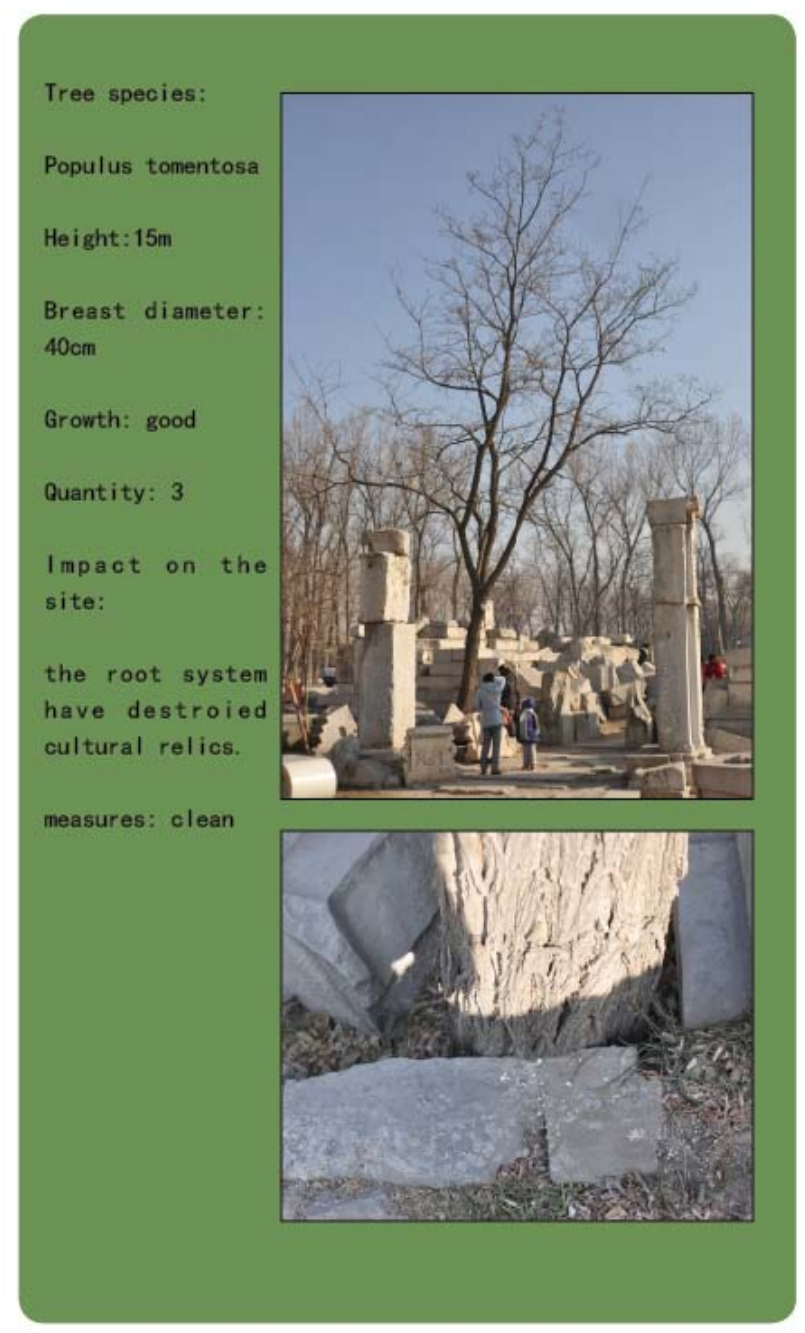

3-2Problems in the Western Mansions 


\subsection{Technical measures and processing methods of the Western Mansions scenic area site}

\subsubsection{Eradicating trees and large shrubs and inhibiting growth}

Considering that most of the vegetation in the park has grown into a tree and its root system is developed, if the root is eradicated directly, it will cause more cracking of the cultural relics. Meanwhile, after the underground root is transplanted, the big pits left may destroy the underground ruins. The standing water, piled debris and rotten leaves and so on may cause possible hazards to the cultural relics. Therefore, before the archaeological excavation work begins, the roots of trees and shrubs should not be directly removed. Instead, the parts above the ground should be cut down manually and, the dead roots system should be kept. In this way, it will cause less interference and destruction of the current situation. The roots systems will not cleaned up until the archaeological excavation work is carried out.(3-3)

For part of the trees and large shrubs that grow near the cultural relics,it will be removed the upper part of the ground , save the root system.So we have not damaged the heritages. But with the continued growth of the root system, it is likely that they will cause damage in the future. For the sake of ecological effects and vegetation protection, they should not be cleaned up but some countermeasures need to taken to curb the growth of the roots. For example, regular pruning of the trunks may be adopted so as to reduce the stems, thereby inhibiting the growth of the plant as a whole and then inhibiting the growth of the lateral roots. During the process of inhibiting growth, physical methods should be used as much as possible, and the chemical reagent should be avoided, because it may damage the cultural relics in the heritage site.

3.2.2Trim the branches and stems of the trees which interfere with or destroy the heritage site:

For part of the vegetation in the vicinity of the heritage site, although the roots have not caused damages to the cultural relics, but their oblique branches may interfere with the heritage site. These branches should be pruned or removed.

For the trees that blocks the way of the tourists: for the vegetation that grows around the boardwalk, its branches may interfere or hinder tourists to visit the heritage site. Thus, the branches below $1.8 \mathrm{~m}$ should be trimmed selectively. The core purpose of trimming is to prevent branches from blocking the way of tourists. Therefore, only the trucks need to be trimmed. There is no need to trim such trees into certain shapes so as not to cause new informational misunderstandings. Meanwhile, in selecting the trimmed branches, it should be done under the onsite guidance of the designers of such green plants.

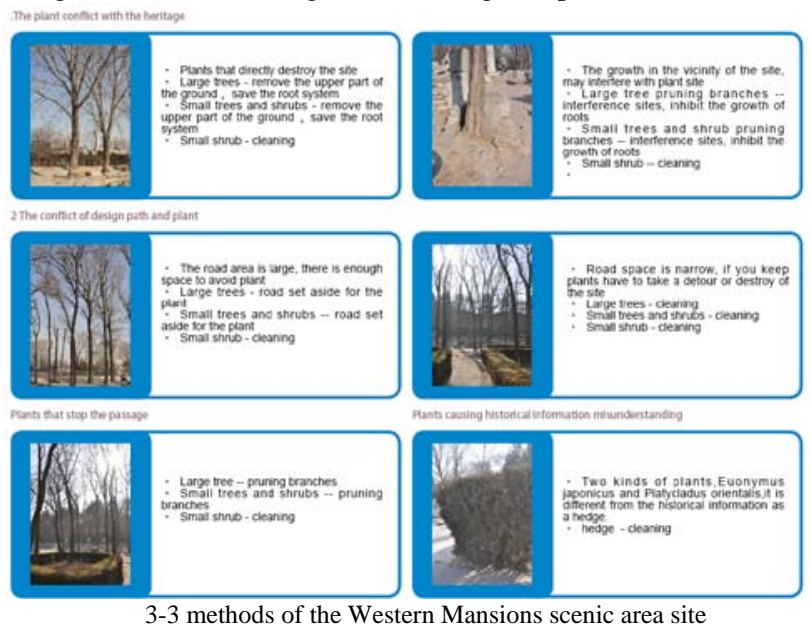

\subsubsection{Clean up the hedges}

At present, the hedges are mainly euonymus japonicus and platycladus orientalis. These two kinds of plants have a shallow root system so that there is no need to excavate. Instead, they need to be removed manually. At the same time, the exposed part of loess, the turf and ground covered plant should be planted.

The replanting of the turf and ground covered plants should be strictly controlled within the scope where the hedges have been cleaned up. Here, the current situation is making everything green by planting plants and so there are no cultural relics left. Therefore, it will not damage the cultural relics. In history, the materials of the turf and ground covered plants are lawns in the Western Mansions scenic area. Thus, it will not lead to any new informational misguidance, either. (3-4)
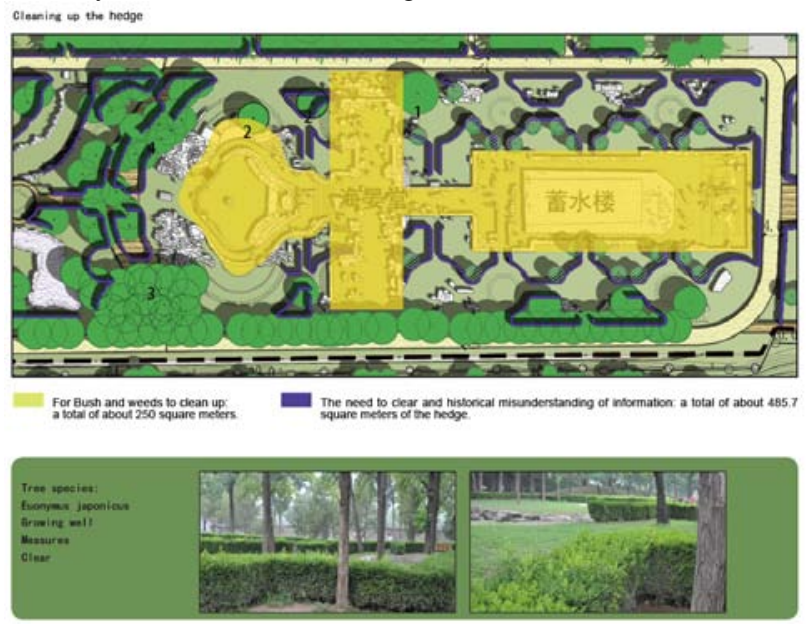

3-4 Clean up the hedges

3.2.4 Cleaning weeds

Weeds should be taken out regularly manually. Different cleaning plans should be made in accordance with the seasons. In spring, the seeds should be cleaned up to prevent them from rooting; in summer, the weeds should be pulled out; and in autumn, the fallen leaves should be cleaned periodically. Try not to use chemical herbicide.

3.2.5 Adjust the design program

Avoid excavation. For the primary consideration of cultural relic protection, the first premise for the display of historical vegetation landscape is not to make any excavation. Therefore, potted plants are the first choice, whose advantages are listed as below:

Firstly, there is no need for excavation, so that the soil of the heritage site will not be destroyed.

Secondly, it helps to distinguish the restored vegetation and other vegetation at the venues, so that visitors can have an intuitive understanding of the types and the layout of the restored plants.

Thirdly, based on the analysis of historical data, in the scenic area, the plants to be trimmed are mainly platycladus orientalis. This kind of plant materials is suitable for trimming and shaping. Besides, their root system is shallow and it is therefore suitable for them to be displayed in the form of potted plant.

Fourthly, through the study of historical data, the author finds, there were records for the potted plants within the scenic area of the Western Mansions. Therefore, in the pots arrangements, the patterns of the historical vessels of planting can be restored, which can display the historical information from another perspective. The patterns of the planting vessels can be divided into two kinds. One is the restored planting vessels in copperplate etchings, which stands for the historical potted plants; the other is the simplified planting vessels to distinguish 
that such plants were planted in the soil but now are displayed in the form of potted plants.

The restored potted plants are arranged only on both sides of the visiting paths, namely the main visiting area for visitors. Such plants are designed along with the wooden trestles and the engineering quantity is so small that there is no need to adjust the current plans. The resorted potted plants along with the wooden trestles will be displayed strictly in accordance with the historical position so that they can exhibit some information of the historical vegetation landscape.

\section{CONCLUSION}

\section{1)Importance:}

Historically, the Western Mansions were an important part of the exchange between Chinese and the Western cultures. It marked the entry of European architecture and art of gardening into Chinese royal gardens. Therefore, the Western Mansions occupy a vital position in garden history of China and even the world. As a complete garden space, plant landscape is an important component of the Western Mansions scenic area that can neither be separated nor ignored. The display value should be placed in the equally important position as the architecture and the water method heritage.

\section{2)Creativity}

Before the Western Mansions scenic areas was established, the tree planting in traditional Chinese gardens followed a core concept: "learning from nature" and "looking natural". The plant pruning is mainly used to cater to the personal aesthetic of the garden owners. It aims only to appreciate the beauty of the trunks of plants and there is no such a method to trim or shape the plants. The plant shape trimming in the Western Mansions scenic areas is the first attempt in traditional garden building history.

3)Uniqueness:

the plant landscape method adopted by the Western Mansions scenic areas integrates the regular lines and array layout of the classic Western gardens with the natural planting layout of traditional Chinese gardens, which are totally different from each other. This is a unique integration in traditional gardens.

4)Feasibility

Because the vegetation is planted naturally and then grows dynamically, the plant landscapes will present different historical appearances in different time nodes. In terms of the location of the planting and the types of the plants, the archaeological work cannot provide more information than the historical record, copperplate engraving or old photos. Therefore, the reappearance of historical vegetation landscape is different from the restoration of the construction and water method heritage site and, it does not have a high sensitivity. Besides, there is no need for it to be carried out after the archaeological excavation.

In accordance with the research findings from existing historical literature, a detailed plant species list at the peak period of the Old Summer Palace can be obtained. In accordance with the records of the copperplate etching, the planting position, types and quantity of the historical vegetation in the Western Mansions scenic areas can be inferred. The contrast and verification of the old photos will provide a strong historical evidence for the reconstruction of the vegetation landscape.

The planning and design of tour routes may constraint and regulate visitors' behaviors. And in the major visiting and travelling areas, the vegetation landscape is reconstructed. In this way, the tourists can experience the vegetation landscape of the Western Mansions scenic area intuitively. Meanwhile, this can reduce the project scale so as not to interfere with archaeological excavation and the main body conservation of the heritage site. (4-1)

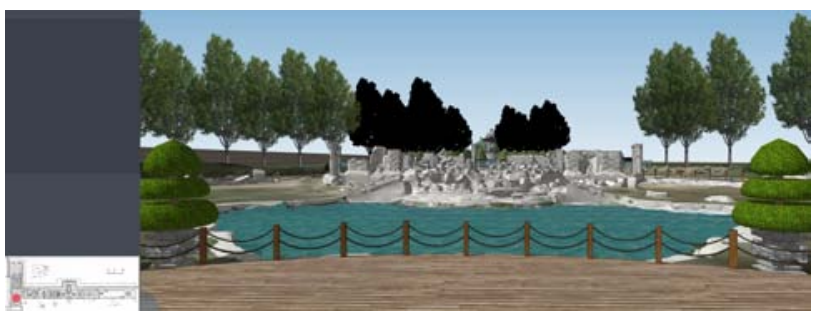

4-1 Pavilion of Delights of Harmony

\section{REFERENCES}

Management related documents:

Chinese Landcape Architecture, 2010, Park Plan, magazine

Chinese Landcape Architecture, history and landcape, 2008, magazine

LandscapeArchitecture,2007. ParkPlan,magazine

Energy Efficient Landscapes , Volume2, Issue2,2014.6

Huazhong Architecture,201107-201206, magazine

The World Heritage in China, 201301-2013.03, magazine

China Cultural Heritage Scientific Reseatch,2013.2

Ministry of Culture of China, 2006. State Administration of Cul tural Heritage introduced.

Ministry of Finance, State Administration of Cultural Heritage, 2005.

World Heritage Division of State Administration of Cultural He ritage,2007. Monitoring: Effective Means for the Protection and Management of World Cultural Heritage, China Cultural Relics News, Page 028, November 23.

The Beauty of Cultural Heritage, Hand drawn works,2014.3

Guo Daiheng,He Yan,Digitization Reproduction Old Summer Palace,2010 